\title{
THE ROOTS ARE HORIZONTAL LADDERS
}

across the path

climbing one rung to the other

my feet find their way

my son is a stranger to me

his eyes are giant pools

of wet stones

I want him to be kind.

How can something so tall

sway and keep its balance?

92

The birch lean together

in their turning shade

I find the urge to

categorize everything

the names of trees and animals

what we mean

to one another

red gooseberry

red of the fruit that opens yellow

cleanly broken under a wheel

red that is swollen and spiny and ripe

Every day you get closer to leaving me

and it is as terrifying

as finding

a cardinal wing 